\title{
Sistemas de recuperación de información adaptados al dominio biomédico
}

\author{
Por Mónica Marrero, Sonia Sánchez-Cuadrado, Julián Urbano, Jorge Morato y José-Antonio Moreiro
}

\begin{abstract}
Resumen: La terminología usada en biomedicina tiene rasgos léxicos que han requerido la elaboración de recursos terminológicos y sistemas de recuperación de información con funciones específicas. Las principales características son las elevadas tasas de sinonimia y homonimia, debidas a fenómenos como la proliferación de siglas polisémicas y su interacción con el lenguaje común. Los sistemas de recuperación de información en el dominio biomédico utilizan técnicas orientadas al tratamiento de estas peculiaridades léxicas. Se revisan algunas de estas técnicas, como la aplicación de Procesamiento del Lenguaje Natural (BioNLP), la incorporación de recursos léxico-semánticos, y la aplicación de Reconocimiento de Entidades (BioNER). Se presentan los métodos de evaluación adoptados para comprobar la adecuación de estas técnicas en la recuperación de recursos biomédicos.
\end{abstract}

Palabras clave: Biomedicina, BioNER, BioNLP, Text-mining, Recuperación de información, Proceso del lenguaje natural, NLP.

Title: Information retrieval systems adapted to the biomedical domain

Abstract: The terminology used in biomedicine has lexical characteristics that have required the elaboration of terminological resources and information retrieval systems with specific functionalities. The main characteristics are the high rates of synonymy and homonymy, due to phenomena such as the proliferation of polysemic acronyms and their interaction with common language. Information retrieval systems in the biomedical domain use techniques oriented to the treatment of these lexical peculiarities. In this paper we review some of these techniques, the incorporation of lexical-semantic resources, and the application of Named Entity Recognition (BioNER). Finally, we present the evaluation methods adopted to assess the suitability of these techniques for retrieving biomedical resources.

Keywords: Biomedicine, BioNER, BioNLP, Text-mining, Information retrieval, Natural Language Processing, NLP.

Marrero, Mónica; Sánchez-Cuadrado, Sonia; Urbano, Julián; Morato, Jorge; Moreiro, José-Antonio. “Sistemas de recuperación de información adaptados al dominio biomédico". El profesional de la información, 2010, mayo-junio, v. 19 , n. 3, pp. 246-254.

DOI: 10.3145/epi.2010.may.04

\section{Introducción y características del dominio biomédico}

El incremento de documentación y la urgencia para localizar respuestas relevantes convierten a la literatura biomédica en área de interés para la aplicación de técnicas de tratamiento textual en el campo de la recuperación de información (IR, information retrieval). Los sistemas de IR en biomedicina (tabla 1) utilizan voca- 


\begin{tabular}{|l|l|l|}
\hline \multicolumn{1}{|c|}{ Motores de recuperación } & \multicolumn{1}{|c|}{ Colección } & \multicolumn{1}{c|}{ Características técnicas principales } \\
\hline $\begin{array}{l}\text { Novoseek } \\
\text { http://www.novoseek.com }\end{array}$ & $\begin{array}{l}\text { Medline, US } \\
\text { Grants, otros }\end{array}$ & $\begin{array}{l}\text { Basadas en diccionarios elaborados automática- } \\
\text { mente }\end{array}$ \\
\hline $\begin{array}{l}\text { PubFocus } \\
\text { http://www.pubfocus.com }\end{array}$ & $\begin{array}{l}\text { PubMed, } \\
\text { Medline }\end{array}$ & $\begin{array}{l}\text { Basadas en diccionarios (NCI Thesaurus y Mouse } \\
\text { Genome database). } \\
\text { Utiliza el índice Journal citation reports en la } \\
\text { ordenación de resultados. }\end{array}$ \\
\hline $\begin{array}{l}\text { BioMedSearch } \\
\text { http://www.biomedsearch.com }\end{array}$ & $\begin{array}{l}\text { PubMed, } \\
\text { Medline }\end{array}$ & Clustering \\
\hline $\begin{array}{l}\text { XplorMed } \\
\text { http://www.ogic.ca/projects/xplormed/info }\end{array}$ & Medline & $\begin{array}{l}\text { NLP (usan TreeTagger para la eliminación de pa- } \\
\text { labras vacías, la desambiguación y normalización } \\
\text { con stemming) }\end{array}$ \\
\hline $\begin{array}{l}\text { Path Binder H (prototype) } \\
\text { http://pathbinderh.plantgenomics.iastate.edu }\end{array}$ & $\begin{array}{l}\text { PubMed, Medline } \\
\text { (parcialmente) }\end{array}$ & $\begin{array}{l}\text { Basadas en diccionarios (Gene \& plant ontology, } \\
\text { Enzyme nomenclature y MeSH). } \\
\text { Incluye filtrado por taxonomía. }\end{array}$ \\
\hline $\begin{array}{l}\text { Textpresso } \\
\text { http://www.textpresso.org }\end{array}$ & $\begin{array}{l}\text { C. Elegans. } \\
\text { Permite añadir } \\
\text { nuevos artículos }\end{array}$ & $\begin{array}{l}\text { Basadas en diccionarios de términos y procesos } \\
\text { elaborados automáticamente a partir del corpus } \\
\text { Caenorhabditis Elegans }\end{array}$ \\
\hline
\end{tabular}

Tabla 1. Ejemplos de motores de recuperación disponibles para información biomédica

bularios controlados (MeSH, Inspec thesaurus, Gene \& plant ontology) para mejorar las búsquedas, siguiendo las propuestas de Salton en los años 60 para los sistemas de IR de propósito general.

Las técnicas de procesamiento de lenguaje natural (NLP, natural language processing), la extracción de información (IE, information extraction) y la minería de datos se han convertido en procesos indispensables para tratar, identificar e inferir información entre la enorme cantidad de datos disponibles. Este conjunto de técnicas, referidas habitualmente como minería de textos (text mining), son utilizadas en los sistemas de IR y adoptan ciertas peculiaridades al ser aplicadas en el área biomédica por las características del dominio y su terminología.

\section{"La normalización de la terminología logra consistencia en el etiquetado y favorece el uso efectivo de herramientas de anotación semántica"}

La terminología biomédica carece de patrones regulares que faciliten la identificación automática de términos, a pesar de que un tercio de sus ocurrencias son variantes, ya sean ortográficas o por permutación, inserción o eliminación (e. g. proteínas Foxp2 y Foxp3) (Jacquemin, 2001). Además, la investigación en biomedicina se caracteriza por estar dividida en subáreas de conocimiento especializadas y conceptualizadas desde diferentes puntos de vista, lo que limita las conexiones entre trabajos (Weeber et al., 2000) y la recuperación cruzada de información. La aparición cons- tante de nuevos términos para un mismo concepto en las diversas áreas y los diferentes registros, así como la proliferación de acrónimos, generan una elevada polisemia y sinonimia léxica, lo que dificulta notablemente la recuperación de información.

Un concepto de este dominio puede tener seis o siete sinónimos porque surgen en áreas diferentes, bien por cuestiones comerciales, por falta de consenso entre los expertos o por evolución u obsolescencia científica (tabla 2).

\begin{tabular}{|l|l|}
\hline 1979-1982 & Immunologic deficiency syndrome \\
\hline 1984-1986 & Human T-cell leukemia virus/HTLV/LAV \\
\hline 1986-1992 & HIV \\
\hline 1992- & HIV-1/HIV-2 \\
\hline
\end{tabular}

Tabla 2. Descriptores de AIDS en MeSH desde su aparición

Por ejemplo, un producto farmacéutico como el paracetamol se conoce también con las denominaciones de DCI o acetaminofén. Su sinónimo en la nomenclatura Iupac (International Union of Pure and Applied Chemistry) es N-(4-hidroxifenil)etanamida, que es equivalente a la fórmula química $\mathrm{C}_{8} \mathrm{H}_{9} \mathrm{NO}_{2}$, y que se representa con el código NO2 BE01 de la ATC (Anatomical, therapeutic, chemical classification system de la $O M S$ ). Además, su nombre comercial varía de un país a otro: en Estados Unidos es conocido con el nombre de Tylenol o Datril, en Inglaterra como Tylex $C D$ o Panadeine, en España Panadol, Termalgin, Efferalgan, Gelocatil o Apiretal, y en Méjico Tempra.

Los acrónimos aumentan el número de sinónimos en la literatura científica, y además se estima que más del $80 \%$ son ambiguos (Liu; Johnson; Friedman, 2002). De hecho, cada cinco artículos en biomedicina 
surge una nueva sigla, que llega a coincidir con un gran número de siglas preexistentes (Spasic; Ananiadou, 2005). La polisemia afecta tanto a nombres comunes (e. g. el término inglés "cold" puede significar "chronic obstructive lung disease", algo "frío" o "resfriado") como a terminología especializada de diferentes áreas de la biomedicina. Por ejemplo, NF2 es el nombre de un gen y de una proteína y una enfermedad relacionada con el mismo (Bodenreider, 2006). NFKB2 hace referencia a proteínas pertenecientes a especies diferentes: la de los humanos y la de los pollos.

\section{Recursos para la representación de conocimiento en biomedicina}

Para afrontar los problemas terminológicos se elaboran sistemas de organización del conocimiento tales como diccionarios, glosarios, clasificaciones, tesauros y ontologías (tabla 3). Sin embargo, esto no siempre simplifica los procesos de indización y recuperación de información. Por ejemplo, el vocabulario controlado Unified medical language system (UMLS), usado con la base de datos bibliográfica Medline proporciona expansión de consultas con términos relacionados. La expansión de la consulta con un vocabulario controlado mejora la efectividad, en especial con la expansión por términos sinónimos (Hersh et al., 2000), pero fenómenos como la polijerarquía pueden disminuir la precisión de los resultados. Por ejemplo, en $\mathrm{MeSH}$ el virus de la inmunodeficiencia humana puede encontrarse bajo los siguientes árboles jerárquicos: RNA virus infections [C02.782], Sexually transmitted diseases [C02.800], Slow virus diseases [C02.839] o Immune system diseases [C20].

http://www.ncbi.nlm.nih.gov/pubmed

\section{"El incremento de documentación y} la urgencia para localizar respuestas relevantes convierten a la literatura biomédica en campo de interés para la aplicación de técnicas avanzadas de IR"

La complejidad de estos recursos es mayor que en otras áreas por el propio conocimiento que debe representarse (por ejemplo, procesos celulares, interacciones entre proteínas, estructuras proteicas, etc.) y la diversidad de áreas a las que pertenece al mismo tiempo. Como consecuencia, los recursos de información en biomedicina resultan difíciles de construir, usar y mantener. A pesar de ello, al menos en biología parece que el entusiasmo por las ontologías ha venido acom- pañado de una general falta de conocimiento acerca de lo que son y de cómo se usan (Soldatova; King, 2005). La iniciativa Open biomedical ontologies (OBO), iniciada en 2001, trabaja para reconducir esta tendencia en el campo de la biomedicina, ofreciendo reglas para la construcción de ontologías en subáreas como anatomía, genómica, proteómica, metabolómica, fenotipos, etc.

\section{http://obofoundry.org}

Otras iniciativas se centran en la construcción de ontologías específicas del dominio de alto nivel, como OBR (Ontology of biomedical reality), utilizada para integrar ontologías de anatomía, fisiología y patología (Rosse et al., 2005). Hay múltiples ejemplos del uso de este tipo de ontologías, como la Molecular biology ontology (MBO) (Schulze-Kremer, 1997) о BFO (Basic formal ontology) y RO (Relation ontology), que dentro del proyecto $O B O$ dan soporte a la construcción de otras ontologías. Por ejemplo, BioTop y ChemTop (Stenzhorn et al., 2008) son dos ontologías de alto nivel para biología y química basadas en ellas. $B F O$ utiliza a su vez dos conocidas ontologías de alto nivel no específicas del dominio: Dolce y SUMO. Dolce es también la base para la ontología biomédica Simple bio upper ontology (SBUO) (Rector; Stevens; Rogers, 2006) y $S U M O$ es la base de la ontología de alto nivel elaborada en el proyecto BioCaster para la vigilancia de enfermedades infecciosas en los países asiáticos (Collier et al., 2007), que integra diferentes fuentes de conocimiento en varios idiomas. Hay además meta-ontologías para el dominio biomédico, como es el caso de Bio-Zen, que unifica diferentes esquemas de representación: Dolce, Simple knowledge organisation system (SKOS), Semantically interlinked open communities (SIOC), Friend of a friend (FOAF), Dublin core y Creative commons (Samwald; Adlassnig, 2008).

En línea con los objetivos de la web semántica, en los últimos años se han producido avances con trabajos como Bio2Rdf y Linking Open Drug Data Project. Estos proyectos ofrecen la posibilidad de integrar esquemas sobre genes, proteínas, medicamentos y ensayos clínicos, tanto sobre otros esquemas específicos de biomedicina como sobre esquemas genéricos (por ejemplo, Dbpedia).

\section{http://bio2rdf.org \\ http://esw.w3.org/topic/HCLSIG/LODD http://dbpedia.org}

Todas estas iniciativas inciden directamente en los resultados de los sistemas de IR y de IE. Dada la complejidad de la terminología biomédica, la aplicación de técnicas basadas en diccionarios en lugar de en sistemas de representación más sofisticados como las ontologías, podría ser una de las razones por las que hasta 


\begin{tabular}{|l|l|l|l|}
\hline \multicolumn{1}{|c|}{ Recurso } & \multicolumn{1}{|c|}{ Dominio } & Contenido & \multicolumn{1}{c|}{ Caracterísicas principales } \\
\hline $\begin{array}{l}\text { UMLS (Unified medical language } \\
\text { system) } \\
\text { http://www.n/m.nih.gov/research/um/s }\end{array}$ & Biomédicos y sanitarios & $\begin{array}{l}>1.000 .000 \\
\text { términos }\end{array}$ & $\begin{array}{l}\text { Utiliza una ontología de alto nivel } \\
\text { para integrar diferentes recursos, } \\
\text { entre ellos Snomed, MeSH y GO }\end{array}$ \\
\hline $\begin{array}{l}\text { Snomed-CT (Systematized } \\
\text { nomenclature of medicine clinical terms) } \\
\text { http://snob.eggbird.eu }\end{array}$ & Historiales clínicos & $\begin{array}{l}400.000 \\
\text { términos }\end{array}$ & $\begin{array}{l}\text { En inglés y español es gratuito, } \\
\text { pero no en alemán. } \\
\text { Implementado en lenguaje OWL. }\end{array}$ \\
\hline $\begin{array}{l}\text { GO (Gene ontology) } \\
\text { http://www.geneontology.org }\end{array}$ & $\begin{array}{l}\text { Términos genéticos, } \\
\text { agrupados por } \\
\text { funciones, procesos y } \\
\text { localizaciones }\end{array}$ & $\begin{array}{l}>9.000 \\
\text { términos }\end{array}$ & $\begin{array}{l}\text { El 50\% de los términos pueden } \\
\text { snapeados en MeSH y }\end{array}$ \\
\hline $\begin{array}{l}\text { MeSH (Medical subject headings) } \\
\text { http://www.nlm.nih.gov/mesh }\end{array}$ & $\begin{array}{l}\text { Biomedicina, incluyendo } \\
\text { enfermería, veterinaria } \\
\text { y sistemas sanitarios }\end{array}$ & $\begin{array}{l}22.995 \\
\text { descriptores }\end{array}$ & $\begin{array}{l}\text { Utilizado para indexación } \\
\text { automática y manual de Medline }\end{array}$ \\
\hline
\end{tabular}

Tabla 3. Ejemplos de sistemas de organización del conocimiento disponibles en el área de biomedicina

ahora se han obtenido peores resultados en biomedicina respecto a otros dominios (Spasic et al., 2005).

\section{"El procesamiento de lenguaje natural (NLP), la extracción de información (IE) y la minería de datos (DM) adoptan ciertas peculiaridades al ser aplicadas en biomedicina"}

\section{Técnicas aplicadas a los sistemas de IR biomédica}

Los sistemas de recuperación de información aplican habitualmente tareas de NLP, como la descomposición del texto en palabras o términos, el etiquetado gramatical, la normalización y, aunque en menor medida, la desambiguación léxica o la resolución de correferencias. La descomposición del texto para identificar los términos debe ser tratada de modo diferente en el dominio biomédico, ya que no puede ser resuelta directamente en base a los espacios en blanco y los signos de puntuación entre palabras (por ejemplo, [3H]R1881 es un único término). Algunos trabajos señalan que los etiquetadores gramaticales adaptados a este dominio mejoran la efectividad (Zhou et al., 2004; Clegg; Sheperd, 2005), lo que justifica la adaptación de las herramientas de NLP para biomedicina. Este es el caso del etiquetador gramatical y semántico Genia, que ha sido entrenado no sólo sobre corpus periodísticos (Wall Street journal corpus) sino también biomédicos (Genia corpus y PennBioIE), permitiéndole trabajar mejor con distintos tipos de documentos biomédicos (Tsuruoka; Tsujii, 2004).

Otra técnica aplicada es la extracción de información, que ha recibido una especial atención en biomedicina (BioNER) durante la última década, principalmente por los nombres de genes y productos genéticos debido al macroproyecto Genoma humano. BioNER se aplica al reconocimiento de ADN, ARN, línea celular, tipo celular, mutaciones, propiedades de las estructuras proteicas, etc.

Los sistemas de BioNER (tabla 4) han seguido una evolución similar a los de dominio general. Han pasado de utilizar técnicas basadas en reglas elaboradas de forma manual, a sistemas basados en aprendizaje supervisado a partir de corpus anotados. Estos últimos, conocidos habitualmente como clasificadores, se basan en la creación automática de reglas a partir de ejemplos anotados en un corpus. En BioNER se utiliza sobre todo este tipo de aproximación, aunque el apoyo de recursos léxicos es más acentuado. El uso de este tipo de recursos proporciona buenas tasas de precisión, pero no de exhaustividad, debido a la constante incorporación de nuevos términos.

\section{"Un concepto en biomedicina puede tener seis o siete sinónimos, y cada cinco artículos surge una nueva sigla que coincide con un gran número de siglas preexistentes"}

Como contrapartida, las técnicas semi-supervisadas son menos frecuentes en BioNER, aunque hay trabajos que utilizan bootstrapping y aprendizaje activo. Esta técnica se aplica en dominios generales desde los años 90 y se basa en la utilización de unos pocos ejemplos iniciales como semillas. A partir de ellos se realiza un aprendizaje progresivo de patrones léxico-sintácticos, basados en las características de las entidades a capturar y su contexto. En el aprendizaje activo, por el contrario, es el propio sistema el que proporciona al usuario los datos con mayor incertidumbre para que los corrija o etiquete, de modo que puedan ser utilizados como nue- 


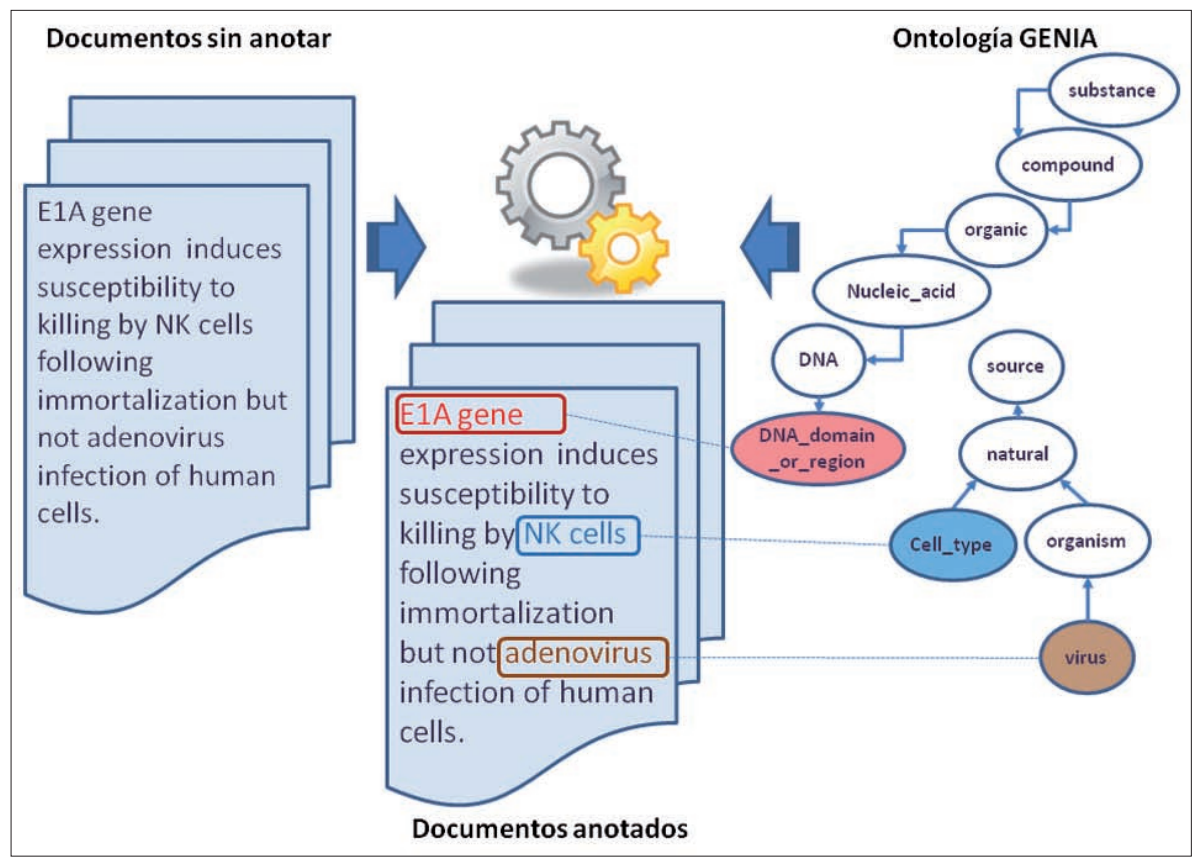

Figura 1. Ejemplo de anotación de un documento con la Ontología Genia detección de relaciones. Tareas como la detección de propiedades funcionales de genes o interacciones de proteínas están adquiriendo especial relevancia. En estas tareas se suman los problemas de BioNER a los múltiples tipos de relaciones diferentes que podemos encontrar. Por este motivo, el apoyo de sistemas de organización del conocimiento es también aquí más necesario que en otras áreas.

\section{Evaluación de la IR en biomedicina}

En las conferencias TREC (Text retrieval conference) se utilizaban colecciones de prueba del dominio médico para la eva- vas semillas. La aplicación de bootstrapping en BioNER es más reciente, y es habitual que tome como semillas corpus etiquetados completos a partir de diccionarios $\mathrm{u}$ ontologías (Morgan et al., 2004; Rong et al., 2009). La carencia de patrones en los términos y la existencia de contextos poco discriminantes por la proximidad semántica de algunas entidades (e. g. los genes se componen de proteínas, lo que hace que compartan procesos) dificultan el aprendizaje progresivo de patrones basado en un reducido número de ejemplos anotados. Por ello, el uso de recursos etiquetados más amplios se convierte en una parte esencial del proceso de aprendizaje.

La aplicación de estos métodos a la Web es poco frecuente, aunque el número de apariciones de ciertos patrones sintácticos se ha utilizado en aprendizaje supervisado (Dingare et al., 2004). En cualquier caso, las tendencias en minería de datos están ligadas a la evolución de la misma Web (Baeza-Yates, 2009). La creciente anotación semántica de la Web con métodos de la web semántica y de la web 2.0 sin duda contribuye a la mejora de los resultados de las técnicas de IE.

Otra de las técnicas de extracción de información frecuentemente aplicadas a los sistemas de IR es la

\section{Bootstrapping}

Literalmente significa correa (strap) de arranque (to boot), abreviado a veces en informática a booting (botar -un sistema operativo-). Este concepto se usa en diferentes áreas (electrónica, negocios, finanzas), y siempre significa poner en marcha algo con pocos medios, partiendo de un inicio muy simple. luación de los sistemas de IR, pero hasta el año 2000 no se creó un track específico para biomedicina. Se midió la capacidad de distintos sistemas para clasificar los documentos de OhsuMed (subconjunto de Medline, con siglas procedentes de Oregon Health Sciences University) con las categorías de $\mathrm{MeSH}$.

En 2003 surgió el Genomics track para la recuperación de documentos relevantes relacionados con genes. En el año 2004 este track se centró en la anotación de genes y proteínas, y se intentó emular el proceso manual que los anotadores del Mouse genome informatics realizan para anotar los genes con $G O$ (Hersh, 2004). La última edición de este track tuvo lugar en 2007, y en él se requería responder a preguntas con entidades cuyo tipo era definido en la propia pregunta (e. g. What [drugs] have been tested in mouse models of Alzheimer's disease?). Otro foro importante en esta área es BioCreative, surgido en 2004 y centrado en el reconocimiento de genes e interacciones entre proteínas. Hay además foros para otro tipo de tareas, como ImageClef para la recuperación de imágenes médicas.

http://www.informatics.jax.org/

http://www.biocreative.org

http://ir.shef.ac.uk/imageclef

"La evaluación de los sistemas de recuperación y extracción de información en biomedicina deben orientarse al usuario, con métricas y métodos capaces de medir su valor en escenarios reales" 


\begin{tabular}{|l|l|l|}
\hline \multicolumn{1}{|c|}{ Herramienta } & \multicolumn{1}{|c|}{ Entidades } & \multicolumn{1}{c|}{ Características principales } \\
\hline $\begin{array}{l}\text { Abner } \\
\text { http://pages.cs.wisc.edu/ bsettles/abner/ }\end{array}$ & $\begin{array}{l}\text { Proteinas, ADN, ARN, } \\
\text { línea y tipo celular }\end{array}$ & $\begin{array}{l}\text { Aprendizaje supervisado (sobre NIpba y } \\
\text { BioCreative). Entrenable. }\end{array}$ \\
\hline $\begin{array}{l}\text { AbGene } \\
\text { http://ftp.ncbi.n/m.nih.gov/pub/tanabe/AbGene }\end{array}$ & Genes, proteinas & $\begin{array}{l}\text { Basada en reglas extraídas estadísticamente } \\
\text { (sobre resúmenes de Medline) }\end{array}$ \\
\hline $\begin{array}{l}\text { PIE } \\
\text { http://pie.snu.ac.kr }\end{array}$ & $\begin{array}{l}\text { Proteinas, } \\
\text { interacciones entre } \\
\text { proteinas }\end{array}$ & $\begin{array}{l}\text { NLP y uso de técnicas basadas en } \\
\text { diccionarios y aprendizaje automático }\end{array}$ \\
\hline $\begin{array}{l}\text { Biorat } \\
\text { http://bioinf.cs.ucl.ac.uk/?id=754 }\end{array}$ & $\begin{array}{l}\text { Proteinas, } \\
\text { interacciones entre } \\
\text { proteinas }\end{array}$ & $\begin{array}{l}\text { NLP y uso de técnicas basadas en } \\
\text { diccionarios y expresiones regulares. } \\
\text { Utiliza el framework de IE GATE. } \\
\text { http://gate.ac.uk }\end{array}$ \\
\hline $\begin{array}{l}\text { Lingpipe } \\
\text { http://alias-i.com/lingpipe/web/download.htm/ }\end{array}$ & $\begin{array}{l}\text { Genes, proteinas y } \\
\text { otros }\end{array}$ & $\begin{array}{l}\text { Herramienta general de IE basada en } \\
\text { aprendizaje supervisado (sobre Genia y } \\
\text { MedPost para biomedicina). Entrenable. }\end{array}$ \\
\hline
\end{tabular}

Tabla 4. Ejemplo de herramientas BioNER disponibles

Las tasas de precisión alcanzadas para tareas de recuperación y extracción de información biomédica se encuentran entre un 70-90\%, mientras que las de exhaustividad rondan el 70\%. Estos resultados suponen un $15 \%$ menos respecto a las tasas alcanzadas en otros dominios como el periodístico (Ananiadou; McNaught, 2006). Sin embargo, las alcanzadas para NER (Named entity recognition) en el dominio periodístico no son superiores. En ocasiones se ha considerado in- cluso superada, con un $90 \%$ de acierto (Cunningham, 2005).

En estas tareas se ha demostrado que el cambio de las fuentes utilizadas, incluso cambiando únicamente el género documental y no el dominio, ocasiona una importante pérdida de efectividad (20-40\%) (Poibeau; Kosseim, 2001). En el dominio biomédico se ha comprobado que entrenar con un corpus anotado y evaluar

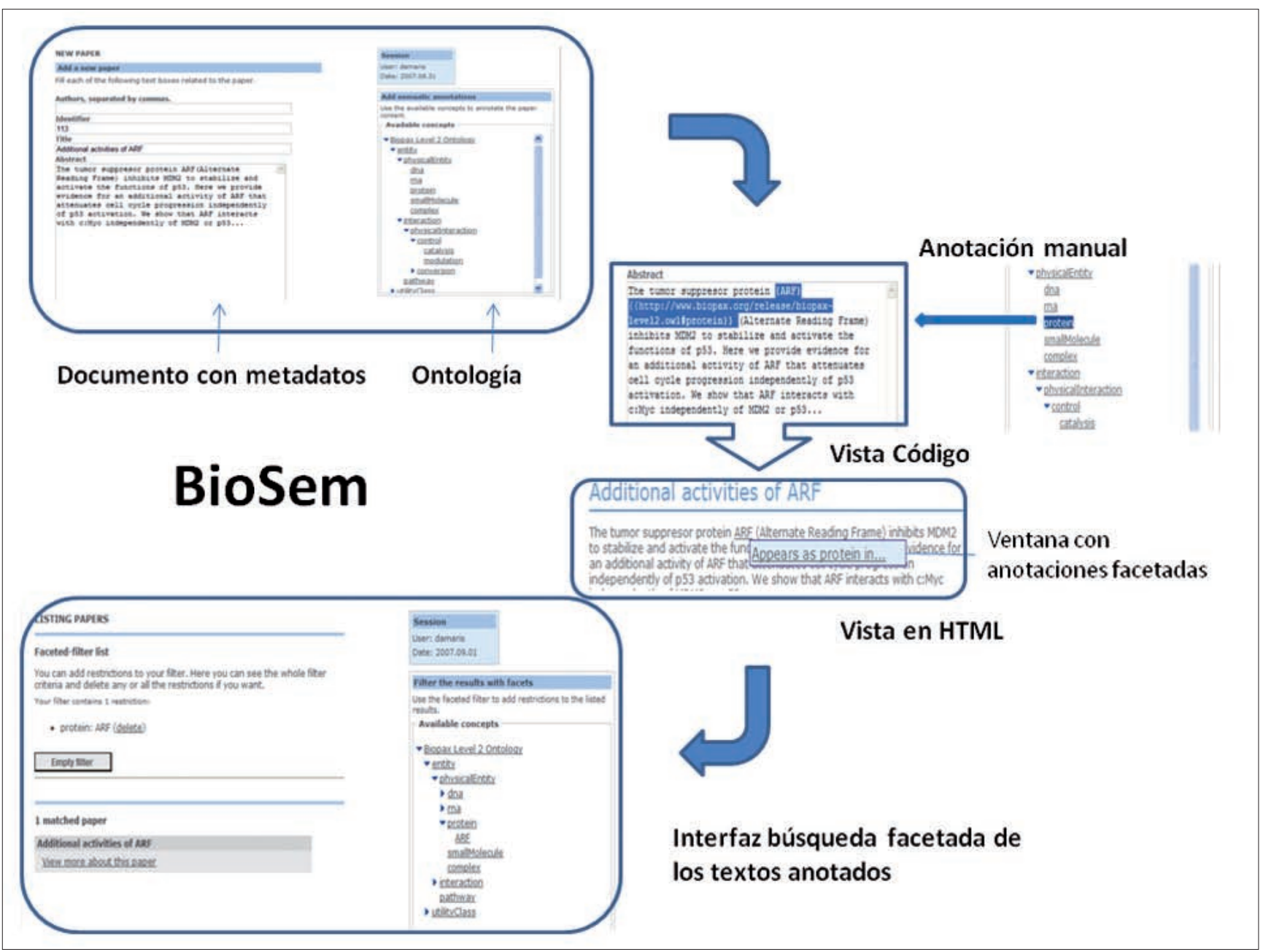

Figura 2. Ejemplo del proceso de anotación mediante la aplicación BioSem (Damaris, Morato y Gómez, 2009) 


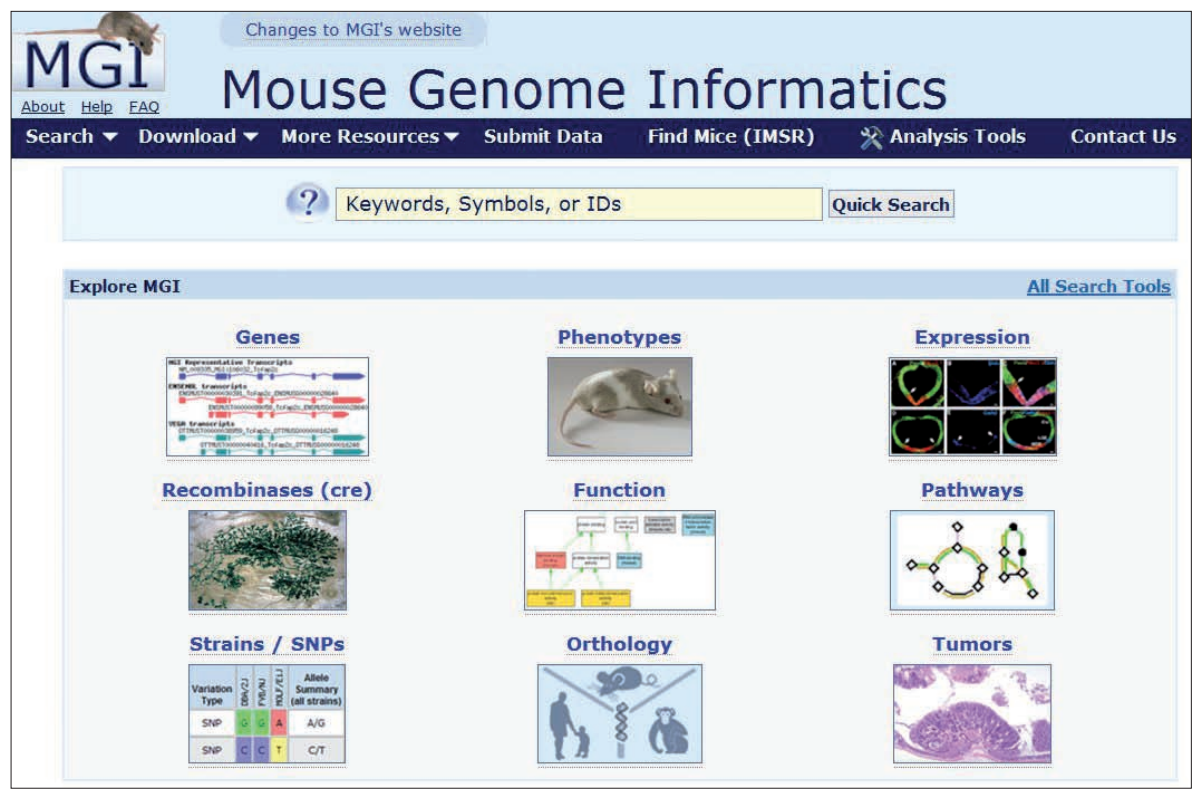

Figura 3. Mouse Genome Informatics, http://www.informatics.jax.org/ ción en biomedicina debe orientarse al usuario, creándose métricas y métodos capaces de medir el valor de tales sistemas en aplicaciones reales (Leser; Hakenberg, 2005; Cohen; Hersch, 2005). Para ello es necesaria la cooperación entre los expertos en recuperación y extracción de información, y los expertos en el dominio de biomedicina. Ejemplos recientes de este tipo de cooperación incluyen el workshop BioCreative 2004 y el Genomics track de TREC, ambos basados en gold standards elaborados por anotadores de bases de datos biológicas en su proceso normal de trabajo. con otro conlleva un descenso del $13 \%$ en la $F$-measure (Leser; Hakenberg, 2005). Dado que las herramientas son entrenadas para colecciones particulares, su comportamiento con otras colecciones difiere, y es de esperar que en las aplicaciones reales sea también diferente. Las características terminológicas, entre ellas la existencia de un gran número de palabras compuestas y la necesidad de conocimientos en diversas subáreas, complican la anotación, con tasas de consenso entre anotadores (inter-annotator agreement) de 75-90\% para genes y proteínas (Gaizauskas et al., 2003). Se hace especial empeño en mejorar la consistencia entre anotadores, por ejemplo con la elaboración de un esquema para la anotación semántica en el dominio de la salud pública (Kawazoe et al., 2009).

En diversos trabajos se sugiere que la evaluación de los sistemas de recuperación y extracción de informa-

\section{"Las características terminológicas y la necesidad de conocimientos en diversas subáreas complican las tareas de anotación de corpus para evaluación"}

\section{Conclusiones}

Son diversas las peculiaridades del dominio biomédico que implican dificultades añadidas para los sistemas de IR. Los problemas de consenso terminológico, tanto en nomenclatura como en organización, y la práctica ausencia de patrones en la terminología utilizada son dos de las más relevantes.

El primer problema influye en la construcción e integración de sistemas de representación de conocimiento y su aplicación en los sistemas de IR. Ante esta situación se impone el uso de internet y la estandarización de los recursos, con formatos y reglas de construcción.

El segundo problema da lugar a un límite en la efectividad de las técnicas basadas en aprendizaje automático. Las iniciativas para normalizar la terminología y las metodologías para lograr consistencia en el etiquetado aportan uniformidad

Figura 4. Biocreative, http://www. biocreative.org 


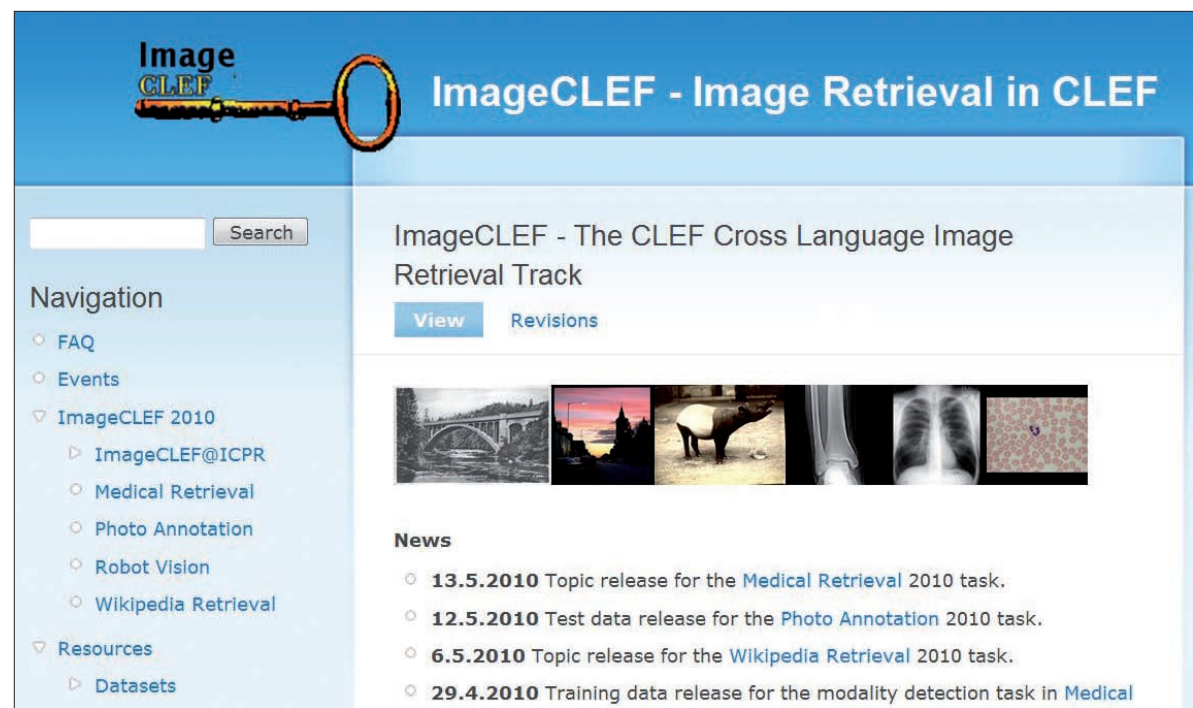

Figura 5. Image Clef, http://ir.shef.ac.uk/imageclef pher; Grover, Claire. "A system for identifying named entities in biomedical text: how results from two evaluations reflect on both the system and the evaluations". En: BioLink meeting at ISMB, 2004.

Gaizauskas, Robert; Demetriou, George; Artymiuk, Pete J.; Willett, Peter. "Protein structures and information extraction from biological texts: the Pasta system". Bioinformatics, 2003, v. 19, n. 1, pp. 135-143.

http://bioinformatics.oxfordjournals.org/ cgi/content/abstract/19/1/135

Hersh, William. TREC genomics track protocol. Oregon Health \& Science University, 2004.

http://ir.ohsu.edu/genomics/2004protocol. html

Jacquemin, Christian. Spotting and discovering terms through natural language processing. Cambridge, MA: MIT Press, 2001, ISBN 0-262-10085-1.

Kawazoe, Ai; Jin, Lihua; Shigematsu, Mika; Bekki, Daisuke; Barrero, Roberto; a la información biomédica y contribuyen a mejorar las tasas de efectividad. Es el caso de The Human Genome Organization (HUGO) con los nombres de genes y proteínas. Estas medidas favorecen además el uso efectivo de las herramientas de anotación semántica como soporte al etiquetado de corpus, lo que supone un medio para lograr una actualización constante de los recursos y proporcionar el soporte que necesitan los sistemas de IR biomédica.

\section{http://www.hugo-international.org}

\section{Bibliografía}

Ananiadou, Sophia (ed.); McNaught, John (ed.). Text mining for biology and biomedicine. Artech House, 2006, ISBN 978-1-58053-984-5.

Baeza-Yates, Ricardo. "Tendencias en minería de datos de la Web". El profesional de la información, 2009, v. 18, n. 1, pp. 5-10.

Bodenreider, Olivier. "Lexical, terminological and ontological resources for biological text mining”. En: Ananiadou, Sophia (ed.); McNaught, John (ed.). Text mining for biology and biomedicine. Artech House, 2006, pp. $43-$ 66, ISBN 978-1-58053-984-5.

http://www.lhncbc.nlm.nih.gov/lhc/docs/published/2006/pub2006007.pdf

Clegg, Andrew B.; Shepherd, Adrian J. "Evaluating and integrating treebank parsers on a biomedical corpus". En: Workshop on software (43rd Annual meeting of the ACL), 2005.

http://citeseerx.ist.psu.edu/viewdoc/download?doi=10.1.1.136.8412\&rep= rep $1 \&$ type $=p d f$

Cohen Aaron; Hersch, William. "A survey of current work in biomedical text mining”. Briefings in bioinformatics, 2005, v. 6, n. 1, pp. 57-71. http://bib.oxfordjournals.org/cgi/content/short/6/1/57

Collier, Nigel; Kawazoe, Ai; Jin, Lihua; Shigematsu, Mika; Dien, Dinh Barrero, Roberto A.; Takeuchi, Koichi; Kawtrakul, Asanee. "A multilingual ontology for infectious disease surveillance: rationale, design and challenges". Language resources and evaluation, 2007, v. 40 n. 3-4, pp. 405-413.

http://naist.cpe.ku.ac.th/downloads/publications/2007_n/Journal_Lecture_ Notes/Multi_Onot_Disease.pdf

Cunningham, Hamish. "Information extraction, automatic". En: Brown, Keith (ed.). Encyclopedia of language and linguistics, v. 1-14, 2nd Edition, Elsevier Science Publishers, 2005, pp. 665-677. ISBN 0-08-044299-4. http://gate.ac.uk/sale/ell2/ie/main.pdf

Dingare, Shipra; Finkel, Jenny; Nissim, Malvina; Manning, Christo-
Taniguchi, Kiyosu; Collier, Nigel. "The development of a schema for semantic annotation: gain brought by a formal ontological method". Applied ontology, 2009, v. 4, n. 1, pp. 5-20.

Leser, Ulf; Hakenberg Jörg. "What makes a gene name? Named entity recognition in the biomedical literature". Briefings in bioinformatics, 2005, v. 6 , n. 4 , pp. 357-369.

Liu, Hongfang; Johnson, Stephen; Friedman, Carol. "Automatic resolution of ambiguous terms based on machine learning and conceptual relatio$\mathrm{ns}$ in the UMLS". Journal of the American Medical Informatics Association, 2002, v. 9, n. 6, pp. 621-636.

McCray, Alexa T.; Browne, Allen C.; Bodenreider, Olivier. "The lexical properties of the Gene ontology (GO)". En: Proceedings of the AMIA symposium, 2002, pp. 504-508.

http://www.lhncbc.nlm.nih.gov/lhc/docs/published/2002/pub2002030.pdf

Morgan, Alexander; Hirschman, Lynette; Colosimo, Marc; Yeh, Alexander; Colombe, Jeff. "Gene name identification and normalization using a model organism database". Journal of biomedical informatics, 2004, v. 37 , n. 6 , pp. $396-410$.

Poibeau Thierry; Kosseim, Leila. "Proper name extraction from non-journalistic texts". Language and computers, 2001, v. 37, pp. 144-157.

Rector, Alan; Stevens, Robert; Rogers, Jeremy. Simple bio upper ontolgy, 2006.

http://www.cs.man.ac.uk/ rector/ontologies/simple-top-bio/

Rong, Xu; Morgan, Alex; Das, Amar K.; Garber, Alan. "Investigation of unsupervised pattern learning techniques for bootstrap construction of a medical treatment lexicon". En: BioNLP workshop, 2009, pp. 63-70. http://aclweb.org/anthology/W/W09/W09-1308.pdf

Rosse, Cornelius; Kumar, Anand; Mejino Jose L. V.; Cook, Daniel L.; Detwiler, Landon T.; Smith, Barry. "A strategy for improving and integrating biomedical ontologies". En: Annual symposium of the AMIA, 2005, pp. 639-643.

http://ontology.buffalo.edu/bio/OBR.pdf

Samwald, Matthias; Adlassnig, Klaus-Peter. "The bio-zen plus ontology". Applied ontology, 2008, v. 3, n. 4, pp. 213-217.

Schulze-Kremer, Steffen. "Adding semantics to genome databases: towards an ontology for molecular biology". En: $5^{\text {th }}$ Int. conf. on intelligent systems for molecular biology, 1997, pp. 272-275.

Soldatova, Larisa N.; King, Ross D. "Are the current ontologies in biology good ontologies?”. Nature biotechnology, 2005, v. 23, n. 9, pp. 1095-1098.

Spasic, Irena; Ananiadou, Sophia. "A flexible measure of contextual similarity for biomedical terms". En: Pacific symposium on biocomputing, 2005, pp. 197-208.

http://helix-web.stanford.edu/psb05/spasic.pdf 
Spasic, Irena; Ananiadou, Sophia; McNaught, John; Kumar, Anand. "Text mining and ontologies in biomedicine: making sense of raw text". Briefings in bioinformatics, 2005, v. 6, n. 3, pp. 239-251

http://bib.oxfordjournals.org/cgi/content/short/6/3/239

Stenzhorn, Holger; Schulz, Stefan; Beißwanger, Elena; Hahn, Udo; Van Den Hoek, László; Van Mulligen, Erik. "BioTop and ChemTop - TopDomain ontologies for biology and chemistry". En: International Semantic Web Conference (Posters \& Demos), 2008, pp. 1-2

http://www.imbi.uni-freiburg.de/ontology/biotop/publications/iswc08.pdf

Tsuruoka, Yoshimasa; Tsujii, Jun'ichi. "Improving the performance of dictionary-based approaches in protein name recognition". Journal of biomedical informatics, 2004, v. 37, n. 6, pp. 461-470.

Weeber, Marc; Klein, Henny; Aronson, Alan R.; Mork, James G.; De Jong-Van den Berg, Lolkje; Vos, Rein. "Text-based discovery in biomedicine: the architecture of the DAD-system". En: AMIA symposium, 2000, pp. 903-907. http://www.lhncbc.nlm.nih.gov/lhc/docs/published/2000/pub2000061.pdf

Zhou, GuoDong; Zhang, Jie; Su, Jian; Shen, Dan; Tan, ChewLim. "Recognizing names in biomedical texts: a machine learning approach". Bioinformatics, 2004, v. 20, n. 7, pp. 1178-1190.

http://bioinformatics.oxfordjournals.org/cgi/content/short/20/7/1178

Mónica Marrero, Sonia Sánchez-Cuadrado, Julián Urbano, Jorge Morato, Jose-Antonio Moreiro. Universidad Carlos III de Madrid.

mmarrero@inf.uc3m.es

ssanchec@ie.inf.uc3m.es

jurbano@inf.uc3m.es

jorge@ie.inf.uc3m.es

jamore@bib.uc3m.es

\section{Máster Oficial Universitario CALSI}

\section{Objetivos}

Especializar a profesionales de la información en la gestión de contenidos a través de diferentes plataformas para todos los ámbitos de la sociedad.

Ahondar y ampliar los conocimientos en Archivística y Documentación con un enfoque dirigido a la aplicación de las tecnologías de la información en sus nuevos canales.

\section{Especialidades}

E-consulting en la sociedad de la información Procesos informativos en las organizaciones. Normativa relativa a los contenidos y su distribución. Sistemas de información en las empresas. Normas y recomendaciones sobre tratamiento y difusión de datos.

\section{Administración electrónica}

Implantación de la administración electrónica desde las oficinas administrativas. Sistematización de los trámites electrónicos y puesta en marcha de un sistema de gestión documental para la administración electrónica a partir de la legislación vigente.

\section{Servicios y contenidos web}

Técnicas aplicadas a la gestión de contenidos en diversos formatos y distribuidos en multiplataforma. Desarrollo de servicios de información en línea.

\section{Estructura del Máster}

75 ECTS a impartir en dos cursos académicos. Horario de tarde

Materias:

42,5 ECTS - se compone de asignaturas comunes y asignaturas de la especialidad escogida

Asignaturas de libre configuración curricular:

\section{7,5 ECTS}

Tesina fin de Máster:

15 ECTS

\section{Profesorado}

Este Máster será impartido por profesorado de diversas universidades nacionales así como profesionales de reconocido prestigio

\section{Plazos orientativos}

Periodo de preinscripción:

Del 17 de mayo al 14 de junio 2010

Periodos de matrícula:

Segunda quincena de julio

Primera quincena de septiembre

\section{Información}

Secretaría del Departamento de Comunicación Audiovisual, Documentación e Historia del Arte (DCADHA) de la UPV.

Teléfono: 963877390

e-mail: dephar@upvnet.upv.es

Página web:

http://www.upv.es/miw/infoweb/po/mas/27/ind ex2005c.html

\section{Preinscripción}

http://www.upv.es/contenidos/PO/menu_4950 $35 \mathrm{c} . \mathrm{html}$

Estos estudios dan acceso al programa de Doctorado. El máster admite estudiantes con titulación universitaria oficial española o de la Unión Europea, así como titulados universitarios de países no pertenecientes a la UE, previa comprobación de la equivalencia del nivel de formación a un título universitario español.

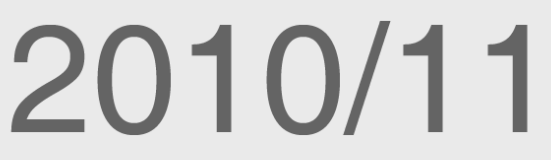

UNIVERSIDAD

POLITECNICA

DE VALENCIA
(1) DCADHA DPTO. DE COMUNICACIÓN AUDIOVISUAL
DOCUMENTACION E HISTORIA DEL ARTE
MINISTERIO

DE EDUCACIÓN Y CIENCIA 